\title{
SHOCK CONDITIONS IN MAGNETIZED JETS
}

\author{
S. APPL AND M. CAMENZIND \\ Landessternwarte Königstuhl \\ D-6900 Heidelberg 1 \\ Federal Republic of Germany
}

\begin{abstract}
The jets in strong radio galaxies (SRG) terminate in hot spots (HS), which are considered as the region where the jet plasma is shocked by interaction with the intergalactic medium. Little is known about the physical parameters in the jets, in particular the velocities. The jump conditions, which are a consequence of conservation of four-momentum, magnetic flux and particle number, provide a means to connect the physical parameters in the jet with those in the HS. The synchrotron radiation which is observed from the HS has a power law spectrum. If the radiating electrons are accelerated by first order Fermi processes, the spectral index in the test particle approximation is dependent on the jump conditions only. These two ingredients, the jump conditions for relativistic MHD shocks, and first order Fermi acceleration, allow to obtain estimates of the jet velocity. It is concluded that the jets in SRG have mildly relativistic velocities of $\simeq 0.3 c$ and fast magnetosonic Mach numbers of $\simeq 3$. Furthermore, the fact that the jets in weaker radio galaxies show no HS is attributed to submagnetosonic velocities. If the shock heats mainly protons, then the postshock plasma heats up to temperatures of a few $10^{11} \mathrm{~K}$. How the protons can be heated and the electrons be accelerated, is subject to the subsequent contribution (Lesch, Appl and Camenzind, these proceedings). In addition, strong magnetic fields reduce the shock strength considerably. Therefore, within the framework of magnetically collimated jets, the VLBI knots are unlikely to be shocks. Details of this work are found in Appl and Camenzind (1988).
\end{abstract}

\section{References}

Appl, S., Camenzind, M.: 1988, Astron. Astrophys. 206, 258 